\title{
C-reactive Protein as a Screening Biomarker in Neonatal Sepsis
}

\author{
Faisal Khan \\ Department of Pediatrics, Aldar Hospital, Madina Munawwara, Kingdom of Saudi Arabia
}

\begin{abstract}
Objective: To measure validity of C-reactive protein (CRP) as screening test for neonatal sepsis (NS) and compare its screening validity between early onset neonatal sepsis (EONS) and late onset neonatal sepsis (LONS).

Study Design: Cross-sectional study

Place and Duration of Study: Neonatal Unit, Town Children Hospital, Peshawar, Khyber Pukhtunkhawa, from August 2016 to February 2017.

Methodology: A total of 385 neonates from age 0 to 28 days with clinical features of neonatal sepsis were sampled using consecutive sampling technique. Two groups were identified, i.e. early onset neonatal sepsis (age $<72$ hours) and late onset neonatal sepsis (age $>72$ hours). Each neonate was sampled for blood culture and C-reactive protein (CRP). Diagnosis of neonatal sepsis was established through a positive blood culture. Data was analysed using SPSS V 25.0. Sensitivity, specificity, negative predictive value (NPV) and positive predictive value (PPV) of CRP was measured and compared in each group.

Results: Analysis showed a low validity of CRP as screening test in neonatal sepsis (Sensitivity $=35.525 \%$, specificity $=58.0 \%$, $P P V=85 \%$ and NPV $=11.83 \%$ ). Initial screening test validity of $C R P$ was low in EONS (sensitivity $=17.16 \%$, specificity $=58.33 \%$, PPV $=72.72 \%$ and NPV=9.81\%) compared to LONS (sensitivity=77.45\%, specificity $=57.14 \%$, PPV=92.94\% and NPV=25.80) Conclusion: CRP as a screening test has low screening validity in early onset neonatal sepsis compared to late onset sepsis.
\end{abstract}

Key Words: Neonatal sepsis, Early onset neonatal sepsis, Late onset neonatal sepsis, C-Reactive protein, Blood culture.

How to cite this article: Khan F. C-reactive protein as a screening biomarker in neonatal sepsis. J Coll Physicians Surg Pak 2019; 29(10):951-3.

\section{INTRODUCTION}

Neonatal sepsis (NS) is the $2^{\text {nd }}$ most common cause of neonatal deaths after prematurity in developing countries. ${ }^{1,2}$ Current neonatal mortality rate in Pakistan is 44 per 1000 live births. ${ }^{3}$ In developing countries like Pakistan, mortality related to neonatal sepsis is three times higher than developed world. ${ }^{4} \mathrm{NS}$ is a systemic infection of new-borns < 28 days of life, with a positive blood culture showing growth of bacteria. NS is classified in to early onset (age of presentation $<72$ hours) and late onset sepsis (age $>72$ hours). ${ }^{5}$

Hematologic markers used to screen neonatal sepsis, include C-reactive protein, absolute neutrophil count, immature/total leukocytes ratio (I:T), and platelets count. Blood culture is the only gold standard test to confirm diagnosis of NS.6,7

C-reactive protein (CRP) is an acute phase protein normally produced by liver within 6 to 8 hours of acute inflammation. Its normal value ranges from 0 to $5 \mathrm{mg} / \mathrm{L}$, (reference range varies in laboratory settings). Among

Correspondence to: Dr. Faisal Khan, Department of Pediatrics, Aldar Hospital, Madina Munawwara, Kingdom of Saudi Arabia E-mail: drfaisalkhanarticle@gmail.com

Received: January 18, 2019; Revised: June 18, 2019;

Accepted: July 04, 2019 inflammatory markers, CRP is a preferable screening tool in initial assessment of NS.8 Moreover, CRP has better screening validity in NS and considered as preferable method to screen a new-born for possible NS. 9,10

The objective of this study was to measure validity of CRP as screening test for possible NS and compare its validity between early and late onset NS rationalising that CRP being non-specific acute phase protein with misleading results before 72 hours of acute neonatal infection due to liver immaturity.

\section{METHODOLOGY}

A cross-sectional study was conducted at Neonatal Unit, Town Children Hospital (TCH), Peshawar, Khyber Pukhtunkhawa from August 2016 to February 2017.

Using WHO software for sample size calculation, anticipated proportion of neonatal sepsis $(P)=(50 \%$, Pakistan Demographic Health Survey 2006-07), confidence level $(\%)=95$, margin of error $(d)=0.05$, standard deviation $(Z)=1.96$ corresponding to $95 \%$ confidence limits, and $\mathrm{n}=\mathrm{Z2P}(100-\mathrm{P}) / \mathrm{E} 2$ (adding 10\% non-response), sample size was determined as $385(p=50 \%)$. Consecutive sampling technique was used to select sample till completion of sample size. Inclusion criteria were neonates from 0 to 28 days of life having clinical features suggestive of NS and willingness of parents to 
Table I: A comparative result of blood culture and C-reactive protein in NS, EONS and LONS.

\begin{tabular}{|c|c|c|c|c|c|c|c|}
\hline \multirow{3}{*}{ C-Reactive protein } & \multicolumn{6}{|c|}{ Blood Culture } & \multirow{3}{*}{ Total (EONS+LONS) } \\
\hline & \multicolumn{3}{|c|}{ Positive } & \multicolumn{3}{|c|}{ Negative } & \\
\hline & EONS & LONS & NS & EONS & LONS & NS & \\
\hline Positive & 40 & 79 & 119 & 15 & 6 & 21 & 140 \\
\hline Negative & 193 & 23 & 26 & 21 & 8 & 29 & 245 \\
\hline Total (EONS+LONS) & 233 & 102 & 335 & 36 & 14 & 50 & 385 \\
\hline
\end{tabular}

Table II: Screening validity of CRP in NS, EONS and LONS.

\begin{tabular}{l|l|r|r}
\hline & NS & EONS & LONS \\
\hline Sensitivity & $35.52 \%$ & $17.16 \%$ & $77.45 \%$ \\
\hline Specificity & $58.0 \%$ & $58.33 \%$ & $57.14 \%$ \\
\hline PPV & $85 \%$ & $72.72 \%$ & $92.94 \%$ \\
\hline NPV & $11.83 \%$ & $9.81 \%$ & $25.80 \%$ \\
\hline
\end{tabular}

participate in study. Excluded patients were those who were advised antibiotics (for any reason) 24 hours before admission to neonatal unit, those positive blood cultures which showed contamination and unwillingness of parents to participate in study. Each neonate was sampled for blood culture and C-reactive protein aseptically. Diagnosis was established through a positive blood culture. CRP value of $>5 \mathrm{mg} / \mathrm{dl}$ was considered positive (as per laboratory indices).

From selected sample $(n=385)$ two groups were identified, i.e. early onset NS (age $<72$ hours) and late onset NS (age $>72$ hours). Data was analysed using SPSS $\vee$ 25.0. In both groups, the validity of C-reactive protein (sensitivity, specificity, negative and positive predictive value) was calculated using $2 \times 2$ table and the results were compared.

\section{RESULTS}

Among 385 neonates, 269 (69.9\%) were grouped in early onset neonatal sepsis and $116(30.1 \%)$ as late onset neonatal sepsis. Overall positive blood cultures were found in $335(87.0 \%)$ and negative in $50(13.0 \%)$ cases. In early onset group, blood culture was positive in $233(86.8 \%)$ and negative in $36(13.4 \%)$ cases, while in late onset group, blood culture was positive in 102 $(87.9 \%)$ and negative in $14(12.1 \%)$.

Results for C-reactive protein (CRP) were positive in 140 (36.4\%) and negative in 245 (63.6\%) neonates. As evident from Table I in EONS group, CRP was positive in $55(20.4 \%)$ and negative in $214(79.6 \%)$ neonates; while in LONS group, CRP was positive in 85 (73.3\%) and negative in $31(26.7 \%)$ neonates.

Table II shows a comparative analysis of screening validity of CRP in early and late onset neonatal sepsis. Results showed CRP had $17.16 \%$ sensitivity in EONS. Specificity of CRP remained $58.33 \%$, while positive and negative predictive values were $72.72 \%$ and $9.8 \%$, respectively; while in late onset neonatal sepsis, CRP had $77.45 \%$ sensitivity, specificity $57.14 \%$, while positive and negative predictive values were $92.94 \%$ and $25.80 \%$, respectively.

\section{DISCUSSION}

Current study was conducted to measure screening validity of CRP as an initial tool for screening NS. In current research, NS was investigated in 385 neonates. Three hundred and thirty-five neonates $(87.0 \%)$ had culture proven NS, while 50 neonates $(13.0 \%)$ had only clinical sepsis. This study concluded a higher percentage of NS compared to other studies concluded by Karamat et al. ${ }^{11}$, Goheer et al. ${ }^{4}$, Mane et al., 9 and Ansari et al. 12 This higher rate was reported due to selection of cases having clinical features of NS. Similar prevalence was also reported by Butt et al.13 Current research showed EONS in 269 neonates $(69.9 \%)$ and LONS in 116 neonates (30.1\%). Similar results were reported by others.4,9,12 A higher rate of EONS compared to LONS was reported due to poor antenatal coverage of high risk deliveries and delayed maternal sepsis screen. ${ }^{14}$

CRP was positive in 140 (36.4\%) cases. In EONS group, $55(20.4 \%)$ neonates had positive CRP; whereas in LONS it remained positive in $85(73.3 \%)$ neonates. Validity of CRP (sensitivity, specificity, positive predictive value, and negative predictive value) in NS (EONS \& LONS) remained $35.52 \%, 58.0 \%, 85.0 \%$, and $11.83 \%$, respectively. In early onset, NS validity of CRP (sensitivity, specificity, positive predictive value, and negative predictive value) as initial screening test remained $17.16 \%, 58.33 \%, 72.72 \%$, and $9.8 \%$, respectively; while in late onset, sepsis validity (sensitivity, specificity, positive predictive value, and negative predictive value) of CRP was $77.45 \%, 57.14 \%, 92.94 \%$, and $25.80 \%$, respectively. This difference in study results were reported by Anwer et al.5, Butt et al. 13, Shirazi et al.6, Vergnano et al. ${ }^{10}$, Naher et al.15, Cetin et al. ${ }^{16}$, Lee et al.17, Kordek et al. ${ }^{18}$ and Hisamuddin et al. ${ }^{19}$

Study results indicate that in comparison to late onset NS, CRP has a low validity as screening biomarker in early onset NS.

The study has certain limitations. Results might be more indicative, if other screening tests like total leukocytes/ Absolute neutrophil count (ANC) also considered as one of the screening biomarkers for NS and results compared accordingly with blood culture. Study would have been improved, if determinants of NS included with comparison drawn between early and late onset sepsis.

\section{CONCLUSION}

Study concluded a low screening validity of CRP as initial septic marker in NS. Moreover, CRP is non-reliable 
test to screen NS before 72 hours of birth; however, it has better screening accuracy in late onset sepsis. In early onset NS CRP should not be used as initial screening test instead of other tests should be relied upon until confirmation of blood culture.

\section{ETHICAL APPROVAL:}

Ethical Approval was taken from Ethical Review Commette of Khyber Medical University before beginning of research work.

\section{PATIENTS' CONSENT:}

Informed consents were taken from the parents (mother or father) of newborn willing to contribute in the study.

\section{CONFLICT OF INTEREST:}

Authors declared no conflict of interest.

\section{AUTHOR'S CONTRIBUTION:}

FK: Abstract, introduction, methodology, results, discussion, analysis and conclusion were written and done.

\section{REFERENCES}

1. World Health Organization. Neonatal and perinatal mortality: Country, regional and global estimates. Geneva: WHO; 2006: 2-5.

2. Zupan J. Perinatal mortality in developing countries. N Engl J Med 2005; 352:2047-8.

3. United Nations Children Fund (UNICEF), demographic indicators. [Available from http//data.unicef.org/country/pak] (Cited on April 7, 2019).

4. Goheer L, Khattak SZ. Early onset neonatal sepsis (risk factors \& clinico-bacteriological profile). Pak Paed J 2014; 38:205-10.

5. Anwer S, Mustafa S. Rapid identification of neonatal sepsis. J Pak Med Assoc 2000; 50:94-8.

6. Shirazi H, Riaz S, Tahir R. Role of the hematological profile in early diagnosis of neonatal sepsis. Ann Pak Inst Med Sci 2010; 6:152-6.

7. Ghosh S, Mittal M, Jaganathan G. Early diagnosis of neonatal sepsis using a hematological scoring system. Indian J Med Sci 2001; 55:495-500.
8. Ahmed E, Rehman A, Ali MA. Validation of serum CRP for the diagnosis and monitoring of antibiotic therapy in neonatal sepsis. Pak J Med Sci 2017; 33:1434-7.

9. Mane AK, Nagdeo N, Thombare VR. Study of neonatal septicemia in a tertiary care hospital in rural Nagpur. JRAAS 2010; 25:19-24

10. Vergnano S, Sharland M, Kazembe $P$, Mwansambo $C$, Heath $P$. Neonatal sepsis: An international perspective. Arch Dis Child Fetal Neonatal Ed 2005; 90:220-4.

11. Karamat KA, Mahmood A, Butt T. Neonatal Sepsis: High antibiotic resistance of the bacterial pathogens in a neonatal intensive care unit in Karachi. J Pak Med Assoc 2002; 52: 348-50.

12. Ansari S, Nepal HP, Gautam R, Shrestha S, Neopane P, Chapagain ML. Neonatal septicemia in Nepal: Early-onset versus late-onset. Int J Pediatr 2015; 2015:379806.

13. Butt TA, Kazir MY, Bhatti MT, Khan HI, Ahmad TM. Evaluation of risk factors and supportive investigations in the diagnosis of neonatal sepsis. Ann King Edward Med Uni 2002; 8:292-4.

14. Puopolo KM, Draper D, Wi S, Newman TB, Zupancic J, Lieberman E, et al. Estimating the probability of neonatal earlyonset infection on the basis of maternal risk factors. Pediatrics 2011; 128:e1155-63.

15. Naher BS, Mannan MA, Noor K, Shahiddullah M. Role of serum procalcitonin and C-reactive protein in the diagnosis of neonatal sepsis. Bangladesh Med Res Counc Bull 2011; 37:40-6.

16. Cetin O, Aydin ZD, Verit FF, Zebitay AG, Karaman E, Elasan S, et al. Is maternal blood procalcitonin level a reliable predictor for early onset neonatal sepsis in preterm premature rupture of membranes? Gynecol 2017; 82:163-9.

17. Lee SY, Park KH, Jeong EH, Oh KJ, Ryu A, Park KU. Relationship between maternal serum $\mathrm{C}$-reactive protein, funisitis and early-onset neonatal sepsis. J Korean Med Sci 2012; 27: 674-80.

18. Kordek A, Toniewska B, Podraza W, Nikodemski T, Rudnicki J. Usefulness of estimation of blood procalcitonin concentration versus C-reactive protein concentration and white blood cell count for therapeutic monitoring of sepsis in neonates. PostepyHig Med Dosw (Online) 2014; 68:1516-23. [Cited on February 14 2019].

19. Hisamuddin E, Hisam A, Wahid S, Raza G. Validity of C-reactive protein (CRP) for diagnosis of neonatal sepsis. Pak J Med Sci 2015; 31:527-31. 